which plays a role in the regulation of cellular proliferation. The most common genetic alteration found in ganglioglioma is BRAF V600E mutation, occurring in $20-60 \%$ of cases; this mutation is also frequently observed in other low-grade neoplasms, including pleomorphic xanthoastrocytoma and pilocytic astrocytoma. Gain of chromosome 7 is also commonly seen. ${ }^{1}$ Other mutations found in a cohort of 40 gangliogliomas using next-generation sequencing included alternative $B R A F$ mutations, $B R A F$ fusion, $K R A S$ mutations, and FGFR1/2 alterations. ${ }^{2}$ The various alterations did not show significant association with disease progression, but analysis was limited by small case numbers.

High-grade transformation of ganglioglioma in the absence of radiation therapy is extremely rare. A review of the literature found 17 cases of malignant transformation of grade I ganglioglioma, with 14 of these describing transformation to anaplastic ganglioglioma (WHO grade III). ${ }^{3}$ Two of the remaining cases described transformation to glioblastoma without additional detail, with the final case showing transformation to anaplastic ganglioglioma, designated as WHO grade IV, with glioblastomatous change of the astrocytic component. Molecular testing in two cases showed BRAF V600E mutation in both the initial and transformed lesions by immunohistochemistry and sequencing. ${ }^{3,4}$ Epithelioid morphology in the transformed lesion was not described in either case. Two cases showed H3F3A (p.K27M) mutations in both lesions, with one case having both $B R A F \mathrm{~V} 600 \mathrm{E}$ and H3F3A mutations. ${ }^{4,5}$ Prognosis amongst the transformed tumours was universally poor with survival ranging from 4 to 42 months. A case series of 54 midline paediatric grade I gangliogliomas found $H 3 K 27 M$ and $B R A F$ V600E mutations in five cases, with one showing progressive disease after 8 years. ${ }^{6}$ Although $H 3 K 27 M$ alterations are required for the diagnosis of diffuse midline glioma WHO grade IV, this case series shows that they are not pathognomonic for, or invariably associated with, poor outcomes in glioneuronal tumours. Mutations in $H 3 F 3 A$ have also been reported in high-grade neuroepithelial neoplasms with glial and dysplastic ganglion cell components. ${ }^{7}$ However, histone $\mathrm{H} 3$ alteration was not demonstrated in our case.

High-grade transformation of $B R A F$-mutant pleomorphic xanthoastrocytoma to $B R A F$-mutant epithelioid glioblastoma has been previously reported, but this has not been documented for $B R A F$-mutant ganglioglioma. ${ }^{8}$ One case report has described an anaplastic ganglioglioma with focal epithelioid cells at diagnosis, with $B R A F$ V600E mutation demonstrated by immunohistochemistry in both components. The authors suggest that the lesion may have eventually evolved into epithelioid glioblastoma, but a temporal relationship was not clearly demonstrated.

The pathogenesis of high-grade transformation of ganglioglioma remains poorly understood due to the paucity of cases in the literature, and relatively few cases detailing molecular associations. Identification of molecular alterations that may be associated with potential high-grade transformation could allow for closer clinical supervision or targeted therapy. This case highlights the potential for malignant transformation of ganglioglioma, in particular to epithelioid glioblastoma, and the need for clinical follow up.

Conflicts of interest and sources of funding: The authors state that there are no conflicts of interest to disclose.
Stephanie Lau, Jonathan Clark, Julie Lokan

Austin Pathology, Melbourne, Vic, Australia

Contact Dr Stephanie Lau.

E-mail: stephanie.lau@austin.org.au

1. Louis D, Ohgaki H, Wiestler O, Cavenee W. World Health Organization Histological Classification of Tumours of the Central Nervous System. 4th ed revised. Lyon: IARC, 2016.

2. Pekmezci M, Villanueva-Meyer JE, Goode B, et al. The genetic landscape of ganglioglioma. Acta Neuropathol Commun 2018; 6: 47.

3. Riesberg G, Bathla G, Gupta S, et al. Malignant transformation and leptomeningeal spread of recurrent ganglioglioma: case report and review of literature. Clin Imag 2018; 48: 7-11.

4. Zanello M, Pages M, Tauziède-Espariat A, et al. Clinical, Imaging, Histopathological and Molecular Characterization of Anaplastic Ganglioglioma. J Neuropathol Exp Neurol 2016; 75: 971-80.

5. Joyon N, Tauziède-Espariat A, Alentorn A, et al. K27M mutation in H3F3A in ganglioglioma grade I with spontaneous malignant transformation extends the histopathological spectrum of the histone H3 oncogenic pathway. Neuropathol Appl Neurobiol 2017; 43: $271-6$.

6. Pagès $\mathrm{M}$, Beccaria $\mathrm{K}$, Boddaert $\mathrm{N}$, et al. Co-occurrence of histone $\mathrm{H} 3$ $\mathrm{K} 27 \mathrm{M}$ and BRAF V600E mutations in paediatric midline grade I ganglioglioma. Brain Pathol 2018; 28: 103-11.

7. Andreiuolo F, Lisner T, Zlocha J, et al. H3F3A-G34R mutant high grade neuroepithelial neoplasms with glial and dysplastic ganglion cell components. Acta Neuropathol Commun 2019; 7: 78.

8. Tanaka S, Nakada M, Nobusawa S, et al. Epithelioid glioblastoma arising from pleomorphic xanthoastrocytoma with the BRAF V600E mutation. Brain Tumor Pathol 2014; 31: 172-6.

9. Murakami C, Yamazaki T, Shintoku R, et al. Anaplastic ganglioglioma with epithelioid cell components. Neuropathology 2018; 38: 498-502.

DOI: https://doi.org/10.1016/j.pathol.2021.09.017

\section{Ovarian mesonephric-like adenocarcinoma: morphological diversity and histogenetic considerations of an unusual tumour}

To the Editor,

Pathologists are familiar with various vestiges of the female apparatus. One of these vestiges, the mesonephric remnants, are frequently encountered in the cervix or the vagina and lesions developing from these remnants, such as mesonephric hyperplasia or adenocarcinoma, are rare but well-recognised. In 2016, McFarland et al. ${ }^{1}$ described a similar type of adenocarcinoma arising in the ovary, and called it mesonephric-like adenocarcinoma (MLA). Since their description, only a few similar cases have been reported, but given the addition of a new chapter in the latest World Health Organization (WHO) classification, more studies are expected to arise, and more information is needed for this rare histological subtype.

A 74-year-old patient was incidentally found to harbour a left ovarian mass. The patient had a family history (daughter) of an ovarian low grade serous carcinoma. Total nonconservative hysterectomy with omentectomy, and peritoneal lesions and lymph node excision was decided. Macroscopic examination revealed a partly solid partly cystic (large cystic space) ovarian left mass measuring $19 \times 14.5 \times 11 \mathrm{~cm}$, 

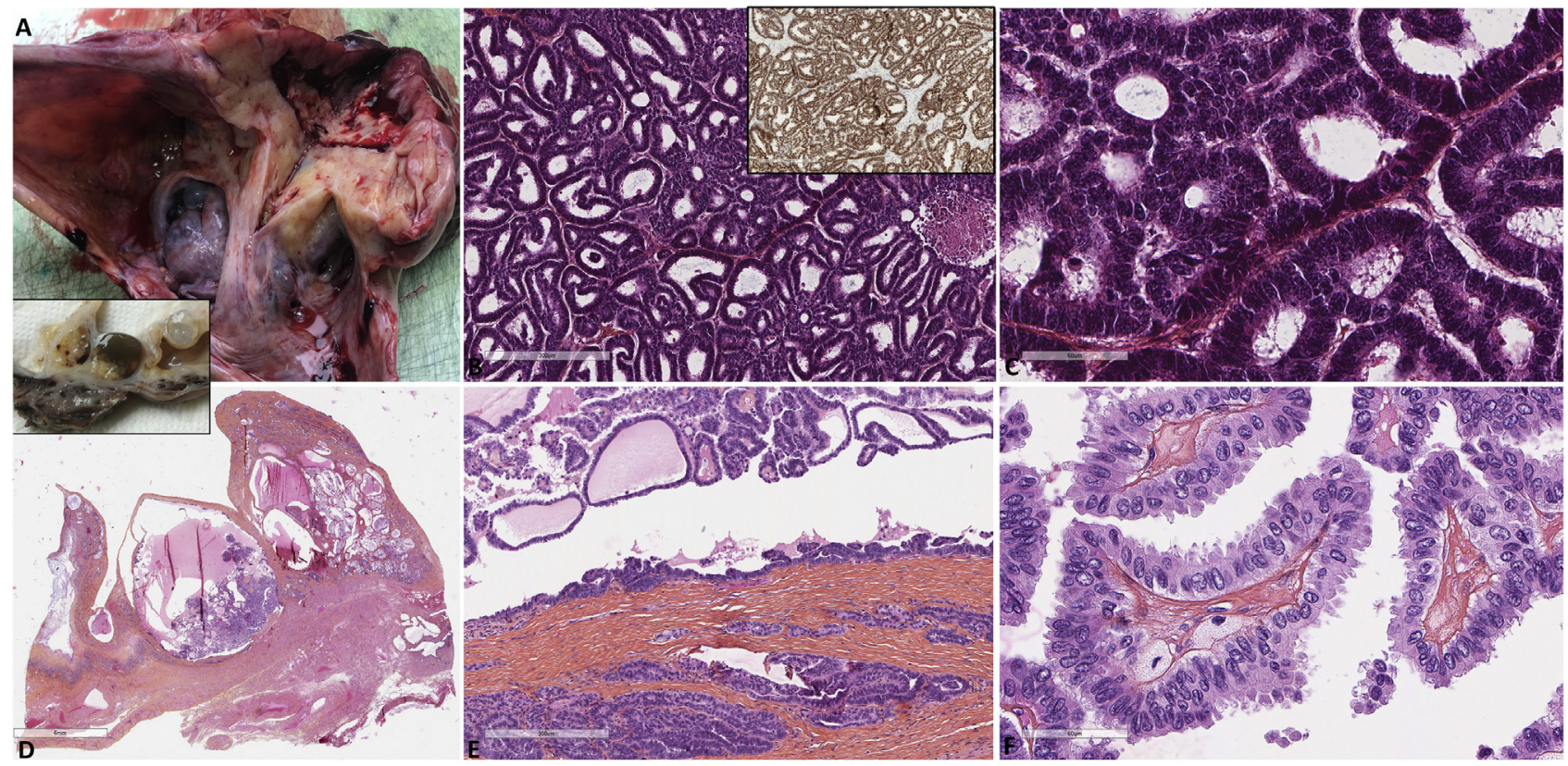

Fig. 1 (A) Macroscopic aspect showing that the tumour is partly cystic, partly solid. (B) The morphology corresponding to the solid area is that of an adenocarcinoma composed of glandular and tubular structures (H\&E; inset: diffuse strong TTF1 expression). (C) Higher magnification of the adenocarcinoma area. (D) Inset: macroscopic aspect of the periphery of the tumour, where smaller cystic spaces often with endocystic excrescences were found. The corresponding slide of this area shows that the intracystic excrescences corresponded to papillary structures, though with less hierarchical branching than the typical serous borderline tumours. Presence of an endometriotic focus (lower right). (E) The intracystic borderline-like component with the mesonephric-like glands at the bottom. (F) The papillae are lined by less atypical cells without significant mitotic count.

weighing $1505 \mathrm{~g}$. Some smaller cystic spaces often containing vegetations were also present (Fig. 1A). Microscopic examination of the solid component showed an adenocarcinoma of glandular, tubular, retiform and papillary architecture composed of moderately atypical cells with numerous mitoses and scant cytoplasm (Fig. 1B,C). The large cystic part of the lesion often showed loss of the lining, with only few residual foci of the same tumour cells recognised. Interestingly, the smaller cystic component had a more variable appearance (Fig. 1D-F): cysts were lined by less atypical cells arranged in papillae resembling a borderline tumour, or by mucinous cells with no atypia and no proliferation, resembling mucinous metaplasia. In these areas (Fig. 2), tubular elements surrounding the cysts frequently showed a luminal eosinophilic material, while glandular and microcystic elements showed larger cells with more abundant eosinophilic cytoplasm,
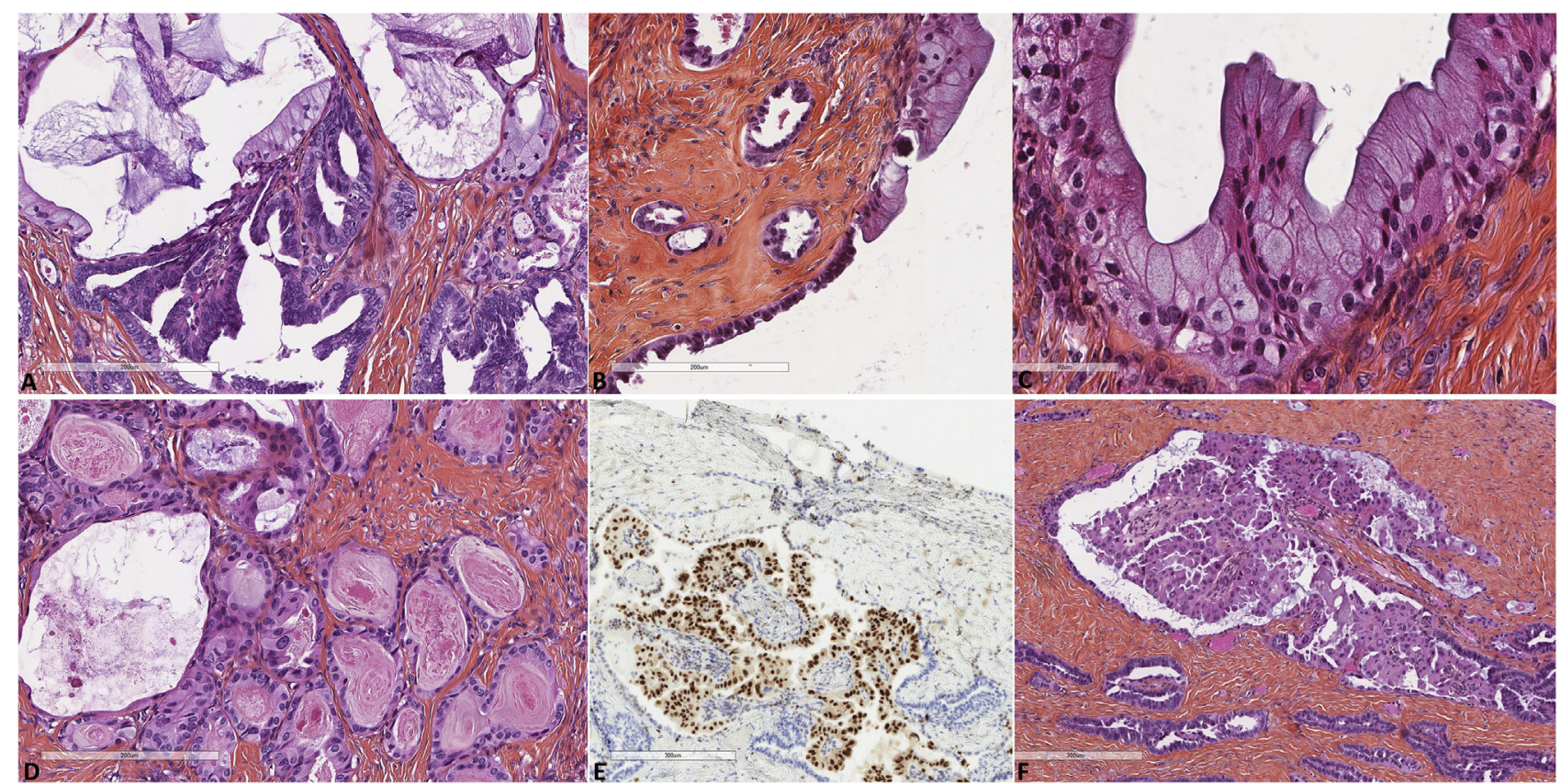

Fig. 2 (A) Also intermixed with the mesonephric-like component, there were mucinous lined cystic spaces. (B) The transition from a more cuboidal (left) to a mucinous lining (right) consistent with mucinous metaplasia. (C) These mucous-secreting cells showed no atypia. (D) Eosinophilic cells and eosinophilic material. (E) ER expression by the focus shown at F. (F) Hobnail cells with eosinophilic cytoplasm and nuclear inclusions. 
compatible with eosinophilic metaplasia. Rare endometriotic foci were found. The immunohistochemical profile was as follows: GATA3+ (mild to moderate patchy expression), TTF1+ (strong and diffuse), CD10+, calretinin-, CK7+ patchy, CK20-, WT1-, PAX8+, ER-, PR-, p53 nonmutated profile, thyroglobulin-. This profile was found in the solid component of the tumour.

In the smaller cystic areas, the following differences were observed: the more eosinophilic glands showed ER expression, while the mucinous component was TTF1-, GATA3 focally + and CK20 focally + but CK7 diffusely + . Also, the borderline-like component was more diffusely GATA3+ and less TTF1+. The tumour was diagnosed as a mesonephriclike adenocarcinoma, TNM stage pT3bN1b and FIGO IIIB. Immunohistochemistry for the four MMR proteins showed retained expression. Next generation sequencing (NGS) was performed in the solid (adenocarcinoma) component and the intracystic papillary borderline-like component revealing KRAS [exon 2 , c. $35 \mathrm{G}>\mathrm{T}$, p. $(\mathrm{Gly} 12 \mathrm{Val})]$ mutation in both, as well as a CTNNB1 mutation [exon 3, c.122 C $>\mathrm{T}$, p.(Thr41Ile)] only in the latter. NGS was further performed for the homologous recombination deficiency genes and the PIK3CA showing no mutations in BRCA1, BRCA2, RAD51C, TP53, PIK3CA, nor large genomic rearrangements for BRCA1, BRCA2, RAD51C. The patient was treated with carboplatin/taxol and is alive and free of disease at 6 months after the initial diagnosis.

Mesonephric lesions of the cervix/vagina are welldocumented; however, their counterpart in the upper female genital tract is rare, essentially represented by the female adnexal tumour of Wolffian origin. It is possible that before the original description of McFarland et al., ${ }^{1}$ similar cases had also been reported, such as the case of Moerman et al. reporting a mesonephric adenocarcinoma of the broad ligament arising in association with a benign lesion, maybe a papillary cystadenoma, ${ }^{2}$ and the case of Ordi et al. 20 years earlier describing a mesonephric carcinoma of the uterine corpus showing no association with the endometrium. ${ }^{3}$ However, the more recent concept of mesonephric-like adenocarcinoma of the ovary or the endometrium probably starts with the report by McFarland et al. ${ }^{1}$ They described five ovarian MLAs showing absence of ER/PR and WT1 for all cases, diffuse positivity for CK7 in all cases, diffuse $(n=4)$ or focal $(n=1)$ positivity for TTF1, diffuse $(n=3)$ or focal $(n=2)$ positivity for PAX8, focal positivity for CD10 (in 2/3 cases studied), focal $(n=1)$ or diffuse $(n=1)$ positivity for GATA3, and wild type p53 expression $(n=3)$. This immunohistochemical profile was further supported by a subsequent study of mesonephric and mesonephric-like adenocarcinomas of the female genital tract, including one ovarian case; however, it is worth noting that almost $40 \%$ of other uterine and cervical carcinomas, especially carcinosarcomas, also showed GATA3 expression, but only rarely TTF1 expression. ${ }^{4}$

In the original report of McFarland et al., the authors suggest a probable mesonephric origin for these neoplasms given their morphology, the absence of squamous and mucinous elements, and the characteristic immunophenotype. ${ }^{1}$ However, the authors do discuss the possibility that these tumours represent Müllerian tumours, especially endometrioid adenocarcinomas differentiating along a mesonephric phenotype, and given the uncertainty, they propose the term MLA for these ovarian and endometrial cases. In a subsequent molecular study of four of the same ovarian cases, a KRAS mutation [p.(Gly12Asp)] was found in all four with additional PIK3CA mutations in two. ${ }^{5}$ In another four ovarian cases, KIT harboured no mutations, despite immunohistochemical cKIT expression; however, the same cases revealed KRAS mutation in all four, and additional CTNNB1 (similarly in exon 3 ) in one and PIK3CA mutations in another. ${ }^{6}$ The presence of $K R A S$ mutations, already known to occur in cervical mesonephric adenocarcinomas, ${ }^{7}$ further reinforced their mesonephric differentiation. However, given their endometrial localisation when uterine tumours, the presence of endometriosis for ovarian cases, the lack of an obvious mesonephric precursor, and the presence of PIK3CA mutations, and despite the lack of squamous/mucinous metaplasia or adjacent atypical endometrial hyperplasia, the authors finally suggest that these ovarian/endometrial neoplasms are likely of Müllerian origin, but display morphological, immunophenotypical and molecular differentiation of mesonephric type.

Shortly after the original report of McFarland et al. ${ }^{1}$ and before the molecular analysis of these tumours, Chapel et al. presented an ovarian mesonephric-like carcinoma combined with low-grade serous carcinoma, each showing distinct morphology and immunophenotype but both harbouring the same NRAS p.(Gln61Arg) mutation, suggesting a clonal relationship and not a collision tumour. ${ }^{8}$ Soon after this observation, McCluggage et al. reported another case of ovarian MLA associated with serous borderline tumour/low grade serous carcinoma, both showing the same KRAS p.(Gly12Asp) mutation despite their individual phenotypes, and describe that in another three of four unpublished cases, a Müllerian lesion (one with borderline endometrioid adenofibroma, one with endometriosis and mixed serous and mucinous cystadenoma, and one with serous cystadenoma) was also present in the same ovary. These findings along with the presence of endometriosis in three of the five ovarian cases originally reported suggested a Müllerian origin for ovarian MLA according to the authors.'

A recent study including 15 ovarian MLAs similarly suggested that 'MLAs are derived from a Müllerian substrate with differentiation along Wolffian/mesonephric lines' and found another component, serous borderline or low-grade, and mucinous borderline, in five cases. ${ }^{10} \mathrm{We}$ believe that the case described here further supports the Müllerian and mostly the endometrioid origin of at least a subset of these tumours. The features described in our case such as the presence of mucinous metaplasia, the presence of a glandular component with more eosinophilic cells often ER + resembling endometrioid adenocarcinomas, and the presence of a CTNNB1 mutation, further support that at least a subset of ovarian adenocarcinomas showing mesonephric morphology and immunoprofile derive from Müllerian epithelium. Indeed, $C T N N B 1$ and PTEN mutations seem to be present only in MLA in comparison to mesonephric carcinomas. ${ }^{10}$

Along with the current case, there seems to exist three ovarian MLAs with a CTNNB1 mutation. ${ }^{6,10}$ Also, we found the CTNNB1 mutation in a component with a more borderline-like architecture without significant atypia and with the same immunophenotype (although more GATA3+, and less TTF1+ cells) that could correspond to the borderline component of this subtype. The phenotypical diversity found in the current case is reminiscent of seromucinous tumours, harbouring mucinous cells, cells with eosinophilic 
cytoplasm, serous-like cells, squamous cells, and similarly considered to derive from an endometrioid linage, leading to deletion of the seromucinous carcinoma ${ }^{11}$ (but not borderline) chapter from the latest WHO classification. Of note, the possible association of ovarian MLAs with microsatellites or HRD gene status is largely unknown; we found no relevant abnormality in the current case.

Finally, given the rarity of these tumours, reports have been limited to only a few cases without considerable follow up, so their clinical course was virtually unknown until recently. However, a recent multicentre, retrospective study gathering 25 ovarian cases (four of which were previously published), ${ }^{4,6}$ among cases from other localisations, revealed a mean age at diagnosis of 61 years (range 36-81), FIGO stage II-IV in almost $40 \%$ of cases and recurrences in $42 \%$ of them, usually distant, often pulmonary, with a 5-year progression-free and overall survival of $68 \%$ and $71 \%$, respectively, implying that these are aggressive tumours. ${ }^{1}$

To conclude, ovarian MLAs should be suspected when an endometrioid morphology is encountered, but with more variable architectures and absence of hormone receptors. GATA3 and TTF1 positivity will then help in the diagnosis. To the best of our knowledge, the features of the current neoplasm have not been reported before: mucinous metaplasia, ER + eosinophilic cells, and a CTNNB1 mutation in a borderline-like component. The morphological, immunohistochemical and molecular features presented here further support the Müllerian, and mostly the endometrioid, origin of a subset of ovarian mesonephric-like adenocarcinomas.

Acknowledgements: The authors would like to thank $\mathrm{Mr}$ Philippe Cosmo from the Tumorothèque/Centre de Ressources Biologiques de CHU Saint-Etienne (BRIF no. BB-0033-00041), as well as Mr Emmanuel Mical for his excellent technical assistance.

Conflicts of interest and sources of funding: The authors state that there are no conflicts of interest to disclose.

Georgia Karpathiou ${ }^{1}$, Celine Chauleur ${ }^{2}$, Tiphanie Picot $^{1}$, Sofia Achour ${ }^{1}$, Thomas Corsini ${ }^{2}$, Mojgan DevouassouxShisheboran $^{3}$, Michel Peoc'h ${ }^{1}$

${ }^{1}$ Pathology Department, University Hospital of SaintEtienne, France; ${ }^{2}$ Gynecology and Obstetrics Department, University Hospital of Saint-Etienne, France; ${ }^{3}$ Pathology Department, Hospices Civils de Lyon, University Claude Bernard Lyon, France

Contact Dr Georgia Karpathiou.

E-mail: gakarpath@yahoo.gr

1. McFarland M, Quick CM, McCluggage WG. Hormone receptornegative, thyroid transcription factor 1-positive uterine and ovarian adenocarcinomas: report of a series of mesonephric-like adenocarcinomas. Histopathology 2016; 68: 1013-20.

2. Moerman P, Amant F, Vergote I. Mesonephric (Wolffian) pseudoendometrioid carcinoma of the broad ligament, arising from a papillary cystadenoma. Int J Surg Pathol 2016; 24: 635-9.

3. Ordi J, Nogales FF, Palacin A, et al. Mesonephric adenocarcinoma of the uterine corpus. Am J Surg Pathol 2001; 25: 1540-5.

4. Pors J, Cheng A, Leo JM, Kinloch MA, Gilks B, Hoang L. A comparison of GATA3, TTF1, CD10, and calretinin in identifying mesonephric and mesonephric-like carcinomas of the gynecologic tract. Am J Surg Pathol 2018; 42: 1596-606.

5. Mirkovic J, McFarland M, Garcia E, et al. Targeted genomic profiling reveals recurrent KRAS mutations in mesonephric-like adenocarcinomas of the female genital tract. Am J Surg Pathol 2018; 42: 227-33.

6. Pors J, Ho J, Prentice L, et al. c-KIT analysis and targeted molecular sequencing of mesonephric carcinomas of the female genital tract. Am J Surg Pathol 2020; 44: 495-502.

7. Mirkovic J, Sholl LM, Garcia E, et al. Targeted genomic profiling reveals recurrent KRAS mutations and gain of chromosome 1q in mesonephric carcinomas of the female genital tract. Mod Pathol 2015; 28: $1504-14$.

8. Chapel DB, Joseph NM, Krausz T, Lastra RR. An ovarian adenocarcinoma with combined low-grade serous and mesonephric morphologies suggests a Müllerian origin for some mesonephric carcinomas. Int J Gynecol Pathol 2018; 37: 448-59.

9. McCluggage WG, Vosmikova H, Laco J. Ovarian combined low-grade serous and mesonephric-like adenocarcinoma. Int J Gynecol Pathol 2020; 39: 84-92.

10. da Silva EM, Fix DJ, Sebastiao APM, et al. Mesonephric and mesonephric-like carcinomas of the female genital tract: molecular characterization including cases with mixed histology and matched metastases. Mod Pathol 2021; 34: 1570-87.

11. McCluggage WG. Endometriosis-related pathology: a discussion of selected uncommon benign, premalignant and malignant lesions. Histopathology 2020; 76: 76-92.

12. Pors J, Segura S, Chiu DS, et al. Clinicopathologic characteristics of mesonephric adenocarcinomas and mesonephric-like adenocarcinomas in the gynecologic tract. Am J Surg Pathol 2021; 45: 498-506.

DOI: https://doi.org/10.1016/j.pathol.2021.09.007

\section{Recurrent plexiform angiomyxoid myofibroblastic tumour (PAMT) of the stomach with aggressive behaviour}

To the Editor,

Plexiform angiomyxoid myofibroblastic tumour (PAMT), also known as plexiform fibromyxoma (PF), is a myofibroblastic tumour, first described by Takahashi et al. in 2007. As per the World Health Organization (WHO) 2018 classification of digestive system tumours, the term plexiform fibromyxoma has been accepted as a distinct entity among the benign mesenchymal tumours of the stomach. ${ }^{2}$ Despite this nomenclature set by WHO, many authors still prefer PAMT as a better terminology as it literally describes the morphology well. As it is a rare and recently described entity, many clinicians and pathologists are unaware of this tumour. Hence, it poses diagnostic difficulties as compared with other mesenchymal tumours of the stomach and most often gets misinterpreted as gastrointestinal stromal tumour (GIST). There has been only one such case report from India. ${ }^{3}$ Our case is the second with many undescribed features which necessitate a fresh insight into its natural history and nomenclature.

A 50-year-old female underwent upper gastrointestinal (GI) endoscopy (July 2017) 1.8 years previously for the complaint of one episode of upper GI bleeding, which demonstrated a $2 \times 2 \mathrm{~cm}$ ulcerated nodular lesion located along the lesser curvature of the body of the stomach (Fig. 1A). Endo-ultrasonography confirmed a hypoechoic lesion arising from the muscularis propria of the gastric wall, suggestive of either GIST or leiomyoma (Fig. 1B). She 